\title{
Editorial
}

\section{Sports and concussion}

Richard G. Ellenbogen, M.D.

Department of Neurological Surgery, University of Washington, Seattle, Washington

The provocative subject of sports-related concussion has captivated the attention of the media, the medical field, and athletes across the US because of the diverse populations affected. This "perfect storm" of events includes career-altering injuries to collegiate and professional athletes, catastrophic outcomes in youth athletes, and blast injuries to soldiers that mimic the biomechanics of sports concussion. ${ }^{?}$

A unifying definition for concussion is elusive simply because the concept has evolved slowly over two millenia, responding only recently to the advances in our scientific knowledge. Hippocrates, in his Corpus, alluded to concussion and, since then, there has been no uniformly accepted definition. The long-lasting contribution from the excellent paper by Duhaime and colleagues ${ }^{2}$ may be to popularize the term "concussion spectrum," which accurately describes the conclusion of this article and our current approach of embracing concussions' protean manifestations. The primary aim of this study was to measure biomechanical factors by means of helmets instrumented with accelerometers and correlate the data with the clinical features of concussion diagnosed in the athletes. The authors have clearly captured the ambiguity of concussion knowledge - the "fog" of current diagnosis and management paradigms is based on very little Class I or II data. This paper is an outstanding attempt by an experienced and innovative team of engineers and clinicians to augment the empirical data that should drive concussion management. It is an ideal model for how sports concussion studies should be executed.

The authors employed no uniform diagnostic criteria, nor did they attempt to influence the criteria used by the diverse medical staff evaluating athletes with suspected concussions, which complicated their analysis. However, the Head Impact Telemetry (HIT, Simbex) technology employed in these helmets is a validated tool consisting of 6 accelerometers distributed in a horizontal plane around the inside of the helmet. Angular acceleration is calculated from the linear accelerations using a proprietary algorithm from the inventors of this tool, who are also authors on this paper. Researchers extract the peak linear acceleration in $\mathrm{x}, \mathrm{y}$, and $\mathrm{z}$ axes and the peak angular acceleration about the $\mathrm{x}$ and $\mathrm{y}$ axes. The HIT System is not able to calculate the angular acceleration about the vertical axis. It is a 5- (not 6-) degree of freedom measurement tool. Despite any shortcomings, it has been tested extensively in a variety of sports environments. This system is intended to provide data that correlate helmet impacts to concussive events in athletes.

Decades ago, concussion diagnosis was equated with loss of consciousness (LOC). Now, the CDC (Centers for Disease Control and Prevention) estimates that less than $10 \%$ of concussions result in LOC. ${ }^{1,4}$ Remarkably, in this paper only $2 \%$ of concussions diagnosed (1 in 48) presented with LOC. In this study, teams depend primarily on self-reporting. This dependence on self-reporting is problematic for any study. Currently, the National Football League has added video instant replay on the sideline and an athletic trainer in the media box to identify suspected concussions and alert medical staff. This independent-observer approach is far superior to athlete self-reporting. Athletes who suffer concussions are not functioning at their cognitive baseline and do not recognize that they have suffered an injury-or simply may not want to be removed from play. One wonders how many concussions were missed in this study, given that $23 \%$ of the diagnoses reported were delayed (average 17 hours) and another $27 \%$ had unspecified onset. The authors' appropriately note that this significant concern may alter the results. Of note, and reassuring to athletes, is that less than $0.01 \%$ (48) of the telemetry-recorded 486,594 helmet impacts resulted in a clinical diagnosis of concussion. Symptoms and signs - as nonspecific as headache, confusion, dizziness, and neck pain-were the most common presentation in the cohort of 44 athletes who suffered a concussion (from the total of 450 athletes from whom data were collected). Missing in Table 1 was the sine qua non of sports concussion, a survey of the neurological examination and the most commonly employed tool in sports concussion medicine, the Sport Concussion Assessment Tool 2 (SCAT 2). This tool, while imperfect, was developed by a seasoned consensus panel in 2009 
and adopted by a variety of sport associations from FIFA (Féderation Internationale de Football Association) to the NCAA (National Collegiate Athletic Association). ${ }^{5}$ Furthermore, an element of this examination, the modified BeSS test, which evaluates the balance of athletes, has been validated and found to be both accurate and sensitive in athletes with concussion. ${ }^{6}$ It was mentioned in the methodology description but not in the results. The fact that the BeSS test was apparently not positive in any of the concussed athletes in this study is highly suspect. Regardless, the baseline testing employed by these investigators was exhaustive and extensive and included neuroimaging, cognitive and neuropsychological tests, and genetic markers. All these baseline tests were repeated after a concussion and at the end of the season: clearly, rigorous methodology. Unfortunately, detailed analysis of these important tests was not included in the paper, making it difficult to fully compare the spectrum of clinical presentations with the biomechanical findings, the principal aim of the study.

Of greatest concern was that one-third of the diagnosed concussions were not associated with a specific collision event. In addition, some contact events associated with concussion in an athlete demonstrated low peak linear and angular accelerations while other athletes with high readings exhibited no clinical symptoms. The explanation of this important observation is manifold. It could be that the HIT tool has issues with biofidelity in certain impact situations. Another hypothesis is that small cumulative impacts may be additive and render an athlete concussed in a delayed fashion. The long-term effects of subclinical impacts are unclear and that hypothesis, although attractive, is difficult to evaluate based on the data presented in this study. ${ }^{3}$ The HIT System may provide accurate correlation of impacts with symptoms in many circumstances, but the large data gaps suggest that we are not ready to abandon clinical judgment and execute onfield decisions using this tool alone.

This study by Duhaime and colleagues is important in that it demonstrates a wide variability in a) presenting symptoms, b) timing of those symptoms, and c) biomechanical correlation with impacts in athletes with concussion. Refining the definition of concussion is perhaps not essential at this juncture despite the authors' legitimate concerns; the broader the definition we adopt, the better-and the more protective for our athletes. Improved biomechanical tools, finite element modeling, and better correlation of biomechanical data with clinical symptoms and signs will likely be forthcoming under the aegis of collaborative research teams like this one. The authors are to be congratulated. Education and advocacy, to include passing the Lystedt Law protecting youth athletes from traumatic brain injury (currently enacted in 35 states), will provide another layer of safety, as important as novel engineering tools. However, in terms of player safety in 2012, there is no replacement for clinical acumen and experience. The sideline medical team needs to recognize a broad "concussion spectrum" as demonstrated so convincingly in this paper. Our student athletes deserve no less.

(http://thejns.org/doi/abs/10.3171/2012.5.JNS12828)

\section{Disclosure}

The author reports no conflict of interest.

\section{References}

1. Cantu RC: Second-impact syndrome. Clin Sports Med 17: 37-44, 1998

2. Duhaime AC, Beckwith JG, Maerlender AC, McAllister TW, Crisco JJ, Duma SM, et al: Spectrum of acute clinical characteristics of diagnosed concussions in college athletes wearing instrumented helmets. Clinical article. J Neurosurg [epub ahead of print October 2, 2012. DOI: 10.3171/2012.8. JNS112298]

3. Gysland SM, Mihalik JP, Register-Mihalik JK, Trulock SC, Shields EW, Guskiewicz KM: The relationship between subconcussive impacts and concussion history on clinical measures of neurologic function in collegiate football players. Ann Biomed Eng 40:14-22, 2012

4. Langlois JA, Rutland-Brown W, Wald MM: The epidemiology and impact of traumatic brain injury: a brief overview. J Head Trauma Rehabil 21:375-378, 2006

5. McCrory P, Meeuwisse W, Johnston K, Dvorak J, Aubry M, Molloy M, et al: Consensus statement on Concussion in Sport-the 3rd International Conference on Concussion in Sport held in Zurich, November 2008. J Sci Med Sport 12: 340-351, 2009

6. Ross LM, Register-Mihalik JK, Mihalik JP, McCulloch KL, Prentice WE, Shields EW, et al: Effects of a single-task versus a dual-task paradigm on cognition and balance in healthy subjects. J Sport Rehabil 20:296-310, 2011

7. Weinberger S: Bombs' hidden impact: the brain war. Nature 477:390-393, 2011

\section{Response}

Ann-Christine Duhaime, M.D.

\footnotetext{
Department of Neurosurgery, Massachusetts General Hospital, Harvard University, Boston, Massachusetts
}

We thank Dr. Ellenbogen for his thoughtful comments. He has been an active advocate for injury prevention nationally and has been in the middle of the maelstrom as this topic has swept into public prominence.

We appreciate his support of the term "concussion spectrum," which we hope may help to bypass potentially distracting debates about specific definitions. At the same time, we advocate for clear characterization of the specific features of a given population under study in concussion research. As Dr. Ellenbogen points out, traumatic brain injury on the milder end of the severity spectrum is a common problem in a number of civilian and military contexts, and there is still a great deal to learn. The goal of the present report was limited to understanding on what basis the diagnosis of concussion was being made under contemporary conditions of play in college teams participating in a larger study on injury biomechanics. The results of these multiyear studies have been or will be published as separate and more detailed analyses of both single and cumulative forces and their clinical and radiological correlates, which will address many of the queries in Dr. Ellenbogen's commentary. What was of interest from the neurosurgical point of view in the present focused analysis was the difference between the features 


\section{Editorial}

most often used to make the diagnosis of concussion in this sports context and those used in the typical emergency department neurosurgical consult, in which a single contact event is followed by an immediate alteration in level of consciousness. Some of the athletes diagnosed with concussion in this study might not have been labeled as concussed by many neurosurgeons-for example, those with only neck pain or delayed onset of headache. What the collaborators on this project learned was that the "concussion spectrum" may be operationally broader than workers in the various fields interested in this topic previously might have thought.

What remains unknown is, in short-what really matters? Does the concussion spectrum "umbrella" contain diverse entities with different consequences-or in some instances, no significant consequences? Do the symptoms matter, or do the forces matter? From a pathophysiological point of view, how do delayed symptoms after multiple contact events compare with single, isolated events associated with immediate loss of consciousness? Are there different profiles for risk of second-impact syndrome, intermediate cognitive dysfunction, or long-term processes-or do these vary among individuals, depending on genetics or other host factors? By carefully characterizing exactly what we are talking about and by continued investigation, which will take more time than the swings of a pendulum, neurosurgeons and others will be best positioned to offer effective treatments and to advocate knowledgeably for appropriate injury-prevention strategies.

Please include this information when citing this paper: published online October 2, 2012; DOI: 10.3171/2012.5.JNS12828. 